\title{
Editorial: The Atmosphere over Mountainous Regions
}

\author{
Miguel A. C. Teixeira ${ }^{1 *}$, Daniel J. Kirshbaum ${ }^{2}$, Haraldur Ólafsson ${ }^{3}$, Peter F. Sheridan ${ }^{4}$ and \\ Ivana Stiperski ${ }^{5}$ \\ ${ }^{1}$ Department of Meteorology, University of Reading, Reading, UK, ${ }^{2}$ Department of Atmospheric and Oceanic Sciences, \\ McGill University, Montreal, QC, Canada, ${ }^{3}$ Department of Physics, University of Iceland, and Icelandic Met Office, Reykjavik, \\ Iceland, ${ }^{4}$ UK Met Office, Exeter, UK, ${ }^{5}$ Institute of Atmospheric and Cryospheric Sciences, University of Innsbruck, Innsbruck, \\ Austria
}

Keywords: downslope winds, horizontal inhomogeneity, hydraulic jumps, large eddy simulation, mountain waves, orographic precipitation, thermally-driven flows, turbulent fluxes

\section{The Editorial on the Research Topic}

\section{The Atmosphere over Mountainous Regions}

Mountainous regions occupy a significant fraction of the Earth's continents and are characterized by specific meteorological phenomena operating on a wide range of scales. Being a home to large human populations, the impact of mountains on weather and hydrology has significant practical implications. Presently, neither the fine-scale structure of orographic precipitation nor the initiation of convection by mountains can be resolved adequately by regional to globalscale models, requiring appropriate downscaling or parameterization. It is likely that orographic precipitation will be affected by non-trivial feedbacks in climate change scenarios.

Mountains modulate the climate and generate atmospheric waves of various scales. Whilst the

OPEN ACCESS

Edited by:

Luis Gimeno,

University of Vigo, Spain

Reviewed by:

Juan Pedro Montávez,

University of Murcia, Spain

*Correspondence:

Miguel A. C. Teixeira m.a.teixeira@reading.ac.uk

Specialty section: This article was submitted to Atmospheric Science, a section of the journal Frontiers in Earth Science

Received: 19 July 2016 Accepted: 26 August 2016 Published: 09 September 2016

Citation:

Teixeira MAC, Kirshbaum DJ, Ólafsson H, Sheridan PF and Stiperski I (2016) Editorial: The Atmosphere over Mountainous Regions. Front. Earth Sci. 4:84. doi: 10.3389/feart.2016.00084 longest mountain waves are adequately resolved in weather prediction models, the shortest still need to be parameterized, as they exert a drag on the atmosphere. This drag not only decelerates the global atmospheric circulation, but also affects temperatures in the polar stratosphere, which control ozone depletion. Locally, strong downslope winds linked to mountain waves (such as the Föhn and Bora) can cause severe damage. Mountain wave breaking in the high troposphere is a source of Clear-Air Turbulence, and large amplitude lee waves, as well as hydraulic jumps, can induce rotors near the surface, which are a major aviation safety hazard.

In this issue, Belušić et al. report a possible occurrence of horizontal roll vortices over the Adriatic Sea during a Bora wind event. Data measured by the National Center for Atmospheric Research Electra aircraft, which sampled the turbulence structure above the Adriatic Sea during the Mesoscale Alpine Program, indicate that horizontal roll vortices with a wavelength of about $1 \mathrm{~km}$ were generated in the strong cold and dry air outbreak associated with the Bora downslope flow.

Using model parameters based on observations taken in the Madeira archipelago, Sachsperger et al. compare several different linear models of two-dimensional interfacial waves developing on a temperature inversion (e.g., the top of the boundary layer), describing how stability in the free atmosphere limits the possible range of lee wavelengths, modulates the length of the stationary wave mode, and strongly constrains the validity of the shallow-water (or long-wave) approximation.

Baines and Johnson theoretically address nonlinear, two-layer, non-Boussinesq, hydrostatic flow with a rigid lid over a long obstacle, where upstream hydraulic jumps can occur, using a new internal hydraulic jump model. Regime diagrams based on the Froude number and the obstacle height show some novel and surprising features, namely in supercritical flow there are situations where hysteresis may occur, with the flow having multiple possible states for the same input conditions.

Mountains induce different types of thermally and dynamically driven circulations (e.g., katabatic and anabatic flows), and affect the boundary layer characteristics and the dispersion of pollutants. Mountains also act to block strongly stratified air layers, leading to the formation 
of gap flows, and cold air-pools (with implications for road safety, pollution, crop damage, etc.).

In this issue, Schmidli and Rotunno analyse the quasisteady-state limit of the diurnal valley wind system over idealized three-dimensional topography, to provide insight into the mass and heat fluxes associated with the along-valley wind. They derive a scaling relation for the quasi-steady-state along-valley mass flux as a function of valley geometry and size, atmospheric stratification, and surface sensible heat flux forcing, and compare it with numerical simulations of the valley wind system.

De Wekker and Kossmann provide a review of the progress made in documenting and understanding the spatio-temporal behavior of dry convective boundary layer (CBL) heights over mountainous terrain. Unlike CBLs over valleys and basins, CBLs over slopes, ridges, or mountain peaks, where advection from multi-scale thermally-driven flows plays an important role, are not well understood, and a universal definition of CBL height over mountains remains unavailable.

Rotach et al. review the role of meso-scale and sub-mesoscale circulations, such as slope and valley winds, in the structure of the boundary layer and turbulent exchanges over complex terrain, with reference to recent idealized numerical simulations. These circulations are ubiquitous in mountainous terrain, develop due to horizontal inhomogeneity in both radiative and dynamical forcing, and are shown to significantly contribute to vertical fluxes of energy and mass between the surface and the atmosphere.

Motivated by its ventilation effect in Munich, Graf et al. investigate Alpine pumping, the thermally driven circulation between the European Alps and the Alpine foreland, using a Regional Climate Simulation to identify the occurrence and describe the spatio-temporal characteristics of this mountainplain wind system, which occurs regularly under clear and calm weather conditions. They estimate the frequency of these circulations, their vertical extent and diurnal variation.

Largeron and Staquet analyse measurements of the atmospheric boundary layer in the Grenoble valleys during persistent inversions in winter, and simulate the most polluted periods using the MesoNH model, focusing on ventilation and flow stagnation effects. They estimate the strength and depth of the inversion, noting that the boundary layer within the inversion layer is decoupled from the synoptic flow, and strongly dependent on the thermal winds, valley topography, and radiative cooling of the ground.

Güttler et al. explore the energetics of the weakly-nonlinear extension to Prandtl's katabatic/anabatic flow model, in terms of the Total Energy (TE) concept, showing that nonlinear effects have a relatively small impact on the variability of the flow solutions, but the maximum of the along-slope wind speed reduces as slope increases. They find that the diffusion, dissipation, and nonlinear interaction terms in the TE budget do not balance perfectly, leading to flow non-stationarity.

Measurement campaigns such as MAP, T-REX, Materhorn, COLPEX, i-Box, provided a wealth of mountain meteorology field data, which is only starting to be explored. Conversely, numerical modeling remains a major method for investigating flow over mountainous terrain. Recent advances in computing power allow numerical simulations of unprecedented resolution, e.g., Large-Eddy Simulations (LES) of rotors, mountain wave turbulence, and boundary layers over mountainous regions.

In this issue, Cuxart discusses the circumstances under which a high-resolution numerical simulations may be classified as LES, noting that aspects such as the complexity of the terrain, and the stability of the atmospheric stratification may render simulations which could be classified as LES in more idealized conditions unable to resolve the inertial sub-range of the turbulence, for example due to the small scale of turbulent eddies in stable stratification.

Rontu et al. use new high-resolution surface elevation data, and new parameterizations of radiation and momentum fluxes, to simulate the weather conditions during the Sochi Winter Olympic Games 2014, using the HARMONIE-AROME-SURFEX numerical model, and validate results against observations from the WMOFROST-2014 program in the Caucasus mountains. Their updated orographic radiation parameterization proves to be crucial to improving the surface temperature forecasts.

Finally, Liu et al. investigate the effects of landfall location and approach angle on track deflection associated with a tropical cyclone passing over idealized and Central Appalachian mountains, using a series of idealized numerical experiments, finding that when the tropical cyclone landfalls on the central portion of the mountain range, its deflection is determined by vorticity dynamics dominated by horizontal vorticity advection, with additional influence from diabatic heating and frictional effects.

Several papers in this special issue were presented at the "Workshop on Advances in Meso- and Micro-meteorology," which took place near Zagreb, Croatia, in November 2015 (http:// www.pmf.unizg.hr/geof/en/research/meteorology/workshop_me so-micrometeology_en).

\section{AUTHOR CONTRIBUTIONS}

All authors listed contributed to this work and approved it for publication.

\section{FUNDING}

MACT acknowledges the financial support of the European Commission, under grant PCIG13-GA-2013-618016. DJK acknowledges the financial support of the Canadian Natural Science and Engineering Research Council, under grant NSERC/RGPIN 418372-12. IS acknowledges the financial support of the Austrian Science Fund (FWF), under grant \#T $781-\mathrm{N} 32$.

Conflict of Interest Statement: The authors declare that the research was conducted in the absence of any commercial or financial relationships that could be construed as a potential conflict of interest.

Copyright (c) 2016 Teixeira, Kirshbaum, Ólafsson, Sheridan and Stiperski. This is an open-access article distributed under the terms of the Creative Commons Attribution License (CC BY). The use, distribution or reproduction in other forums is permitted, provided the original author(s) or licensor are credited and that the original publication in this journal is cited, in accordance with accepted academic practice. No use, distribution or reproduction is permitted which does not comply with these terms. 